\title{
Acute flaccid paralysis following enterovirus D68 associated pneumonia, France, 2014
}

M Lang ${ }^{1}$, A Mirand (amirand@chu-clermontferrand.fr) ${ }^{2,3}$, N Savy'1 ${ }^{1}$ C Henquell ${ }^{2,3}$, S Maridet $^{1}$, R Perignon ${ }^{4}$, A Labbé ${ }^{1}$, H PeigueLafeuille ${ }^{2,3}$

1. CHU Clermont-Ferrand, NHE, Service de réanimation pédiatrique, Clermont-Ferrand, France

2. CHU Clermont-Ferrand, Laboratoire de Virologie, Centre National de Référence des Enterovirus/Parechovirus - laboratoire associé, Clermont-Ferrand, France

3. Université d'Auvergne, EA4843 Epidémiologie et pathogénie des infections à entérovirus, Clermont-Ferrand, France

4. CHU Clermont-Ferrand, NHE, Département d’imagerie pédiatrique, Clermont-Ferrand, France

Human enterovirus D68 (EV-D68) is known to be associated with mild to severe respiratory infections. Recent reports in the United States and Canada of acute flaccid paralysis (AFP) in children with detection of EV-D68 in respiratory samples have raised concerns about the aetiological role of this EV type in severe neurological disease. This case study is the first report of AFP following EV-D68 infection in Europe.

We report the first case of acute flaccid paralysis (AFP) following enterovirus-D68 (EV-D68) infection in Europe. The United States (US) and Canada are currently experiencing nationwide outbreaks of EV-D68 infections associated with severe respiratory diseases especially in children with underlying respiratory disease that began in mid-August 2014 [1,2]. Concomitantly, clusters of neurological illness characterised by AFP with anterior myelitis have been reported in the US and Canada [3,4]. The detection of EV-D68 in nasopharyngeal specimens of some affected children raises the question of a possible link between EV-D68 infections and severe neurological disease.

Human enterovirus D68 belongs to the enterovirus D species within the Enterovirus genus in the Picornaviridae family. Biologically close to rhinoviruses, EV-D68 has been mainly associated with acute respiratory infection with clinical presentation ranging from mild to severe disease requiring intensive care [5-11].

\section{Case report}

The patient was a previously healthy four year-old boy who was initially taken to his general practitioner's surgery for headache and vomiting on 20 September 2014 . On 22 September 2014, he presented with a febrile meningeal syndrome without any sign of encephalitis.

Cerebrospinal fluid (CSF) showed pleocytosis (190 leucocytes: $92 \%$ of lymphocytes, norm<10 leucocytes)

\section{FIGURE 1}

Spinal magnetic resonance image, acute flaccid paralysis case following enterovirus D68 infection, France, 2014

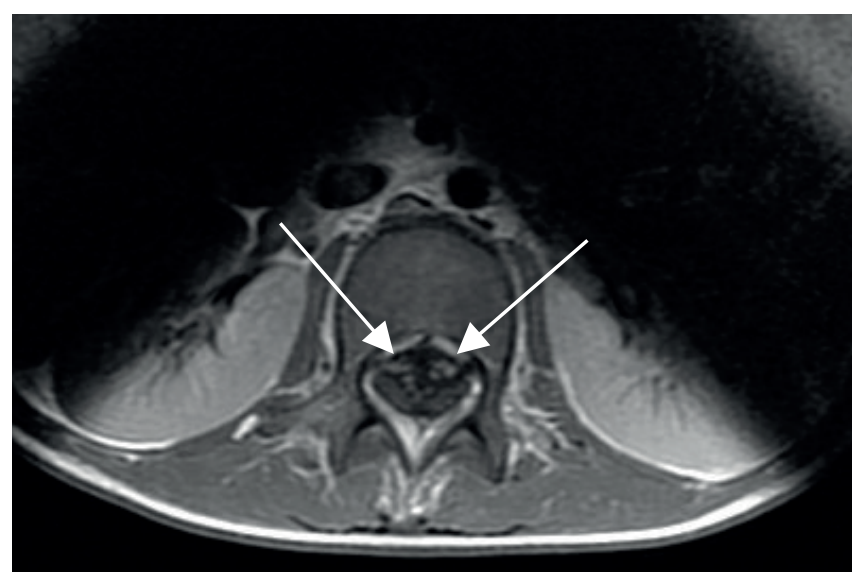

Gadolinium enhancement of the ventral nerve roots of the cauda equina is shown (arrows).

with normal protein and glucose levels, consistent with aseptic meningitis. On 26 September, he was transferred to the paediatric Intensive Care Unit for mechanical ventilation and fluid restoration because of acute respiratory distress and haemodynamic failure. Chest X-ray and thoracic tomodensitometry (TDM) confirmed bilateral pneumonia. Intravenous antibiotic therapy (ceftriaxone) was initiated. Acute myocarditis with apical hypokinesia was assessed by ultrasound examination. Levels of $\mathrm{N}$-terminal of the prohormone brain natriuretic peptide (NT proBNP) and troponine Ic were up to $2,925 \mathrm{ng} / \mathrm{L}$ (norm: $<450 \mathrm{ng} / \mathrm{L}$ ) and $3.06 \mu \mathrm{g} / \mathrm{L}$ (norm: $<0.045 \mu \mathrm{g} / \mathrm{L}$ ), respectively. Leucocytes were elevated two-three times above the upper limit of normal $\left(20,630 / \mathrm{mm}^{3}\right.$, norm: $\left.4,500-13,000 / \mathrm{mm}^{3}\right)$ with $16,880 / \mathrm{mm}^{3}$ of polynuclear cells (norm: $1,500-8,000 /$ $\left.\mathrm{mm}^{3}\right)$. C-reactive protein level was elevated $(75.8 \mathrm{mg} / \mathrm{l}$, norm: $<10 \mathrm{mg} / \mathrm{l})$. The patient received $0.5 \mathrm{~g} / \mathrm{kg} /$ day of 


\section{FIGURE 2}

Phylogeny of enterovirus D68 sequences inferred with 190 VP1 sequences
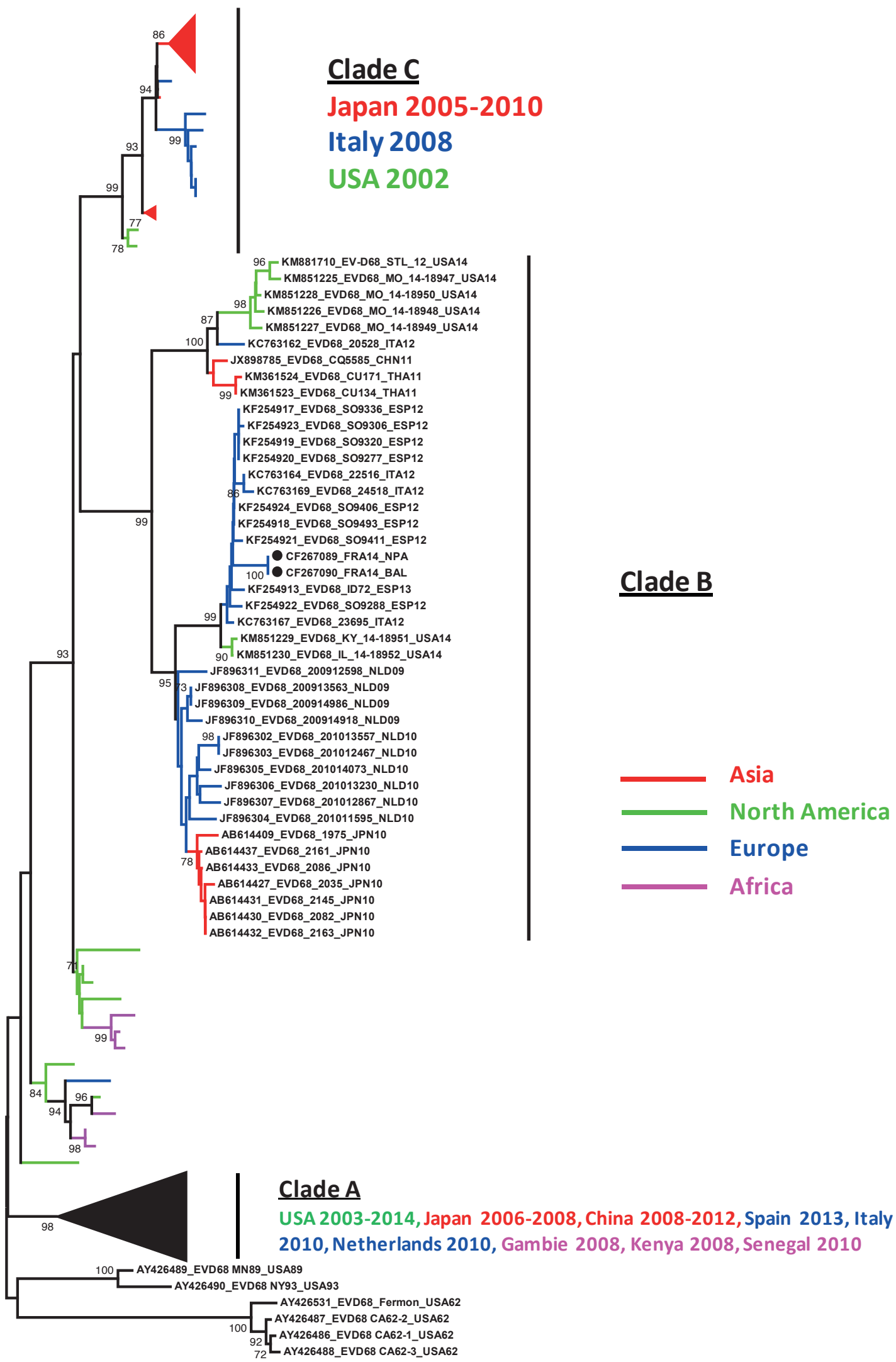

The phylogenetic tree was constructed by the neighbor-joining method and evaluated with 1,00o bootstrap pseudoreplicates, using MEGA5. Only bootstrap values > $70 \%$ are indicated. Genetic distances were calculated with Tamura-Nei's model of evolution and branch length is drawn to the indicated scale (proportion of nucleotide substitutions per site. Sequences were $740 \mathrm{pb}$ long and started to nucleotide 1 relative to the VP1 gene of the Fermon prototype strain. For clarity, taxon names are not fully included in the tree except for clade B. The strains identified in the nasopharyngeal aspirate (CF267089_FRA14_NPA) and in the bronchoalveolar fluid (CF267089_FRA14_BAL) are labeled with a filled circle. Geographical origins and time of isolation of strains are indicated by the ISO-code abbreviation followed by the year of isolation. 
intravenous immunoglobulin (IVIG) and milrinone for four days. On 27 September, he presented with flaccid tetraparalysis and dysphagia. Cerebral magnetic resonance imagery (MRI) was normal but spinal MRI showed gadolinium enhancement of the ventral nerve roots of the cauda equina (Figure 1 ).

Somatosensory evoked potentials confirmed that only the motor pathway was affected. Acute polyradiculoneuritis was excluded because there was no albumino-cytological dissociation and no antiganglioside antibodies in the CSF. There was no paraneoplasic syndrome (whole body-TDM and biological tumour markers were negative) and no inflammatory disease. Plasmapheresis and IVIG were implemented to shorten the recovery period. As of 6 november, the child has only recovered partial mobility of the extremities and of his left arm.

The child had up-to-date immunisation against poliomyelitis. He had neither underlying respiratory illness nor previous history of chronic disease, immunodeficiency or tick exposure. He had not travelled recently outside France and had had no contact with anyone arriving from North America. No family member presented with respiratory symptoms.

Blood, pre-IVIG serum, urine, respiratory and stool samples were screened. Bacteriological investigations including cultures, serology and genome detection in blood and CSF yielded negative results (Table).

Virological screening consisting in viral genome detection for numerous neurotropic viruses including EVs of three consecutive CSF was negative, as were all serological tests (Table). Viral cultures were negative. Rhinovirus-EV genome was detected in nasopharyngeal aspirates, bronchoalveolar fluid (BAL) and a stool sample using a one-step RT-PCR with previously described primers targeting the $1 \mathrm{~A}$ and $1 \mathrm{~B}$ regions encoding the $\mathrm{VP}_{4}-\mathrm{VP}_{2}$ capsid proteins [12]. To distinguish between rhinoviruses and EVs, amplified products were subjected to direct sequencing as previously described [13]. Blast analysis confirmed by phylogenetic analysis with VP4-VP2 sequences of rhinovirus and EV prototype strains assigned the strains to EV-D68. Partial 1D gene encoding the VP1 capsid protein was amplified by semi-nested RT-PCR using EV-D68 specific primers described by Tokarz et al. [14] in respiratory and stool samples, and subsequently sequenced (accession number LN626610). Phylogenetic investigation with all available sequences of EV-D68 (as of 9 October 2014) indicated that the strains belonged to clade $B$, according to the classification previously described [14]. The $\mathrm{VP}_{1}$ sequences were genetically close to sequences of some of the EV-D68 strains detected in 2014 in the United States (Figure 2).

\section{Discussion}

While EV-D68 has to date been almost exclusively associated with respiratory diseases, investigations are currently underway to determine its role in the acute neurological illnesses that have been reported in children in the US [3] and in Canada [4] since August 2014. Nine EV-D68-associated deaths are currently being investigated at the US Centers for Disease Control and Prevention (CDC) to confirm or refute EV-D68 as the cause of death [15]; as of 5 November, no information has been released about the death's preceding symptoms. The case reported here meets the definition given by CDC to identify similar neurological manifestations characterised by acute onset of focal limb weakness occurring on or after 1 August 2014 and MRI showing a spinal cord lesion largely restricted to grey matter [16]. Common features with the cases reported in the US include (i) respiratory illness preceding development of neurological symptoms, (ii) a local epidemiological context of EV-D68 detection among children admitted to hospital for respiratory infections leading to asthma crisis (data not shown) and (iii) EV-D68 detection in respiratory samples. By contrast, to our knowledge, neither meningeal syndrome nor myocarditis and acute respiratory distress syndrome had been reported in the days preceding the onset of paralysis in the US patients. The enterovirus genome was not detected in the CSF of this patient and we cannot assert that EV-D68 was associated with meningitis. There are two case reports in the literature of EV-D68 infection associated with severe neurological disease as evidenced by detection in the CSF $[17,18]$. As in recent reports, the significance of EV-D68 association with AFP is hampered by the fact that it was only detected in respiratory or stool samples, in which enteroviruses can be detected many weeks after infection. However, the absence of detection in CSF does not necessarily rule out this possibility since poliovirus and EV-A71, two recognised neurotropic EVs, are not frequently recovered [19]. Further physiopathological studies may be needed to assess the neurotropism of EV-D68.

There are increasingly numerous reports of polio-like illnesses in the US (64 cases as of 30 October 2014) [15]. Surveillance of AFP cases has already been implemented as a measure in the global initiative to eradicate poliomyelitis and should allow rapid identification of similar neurological manifestations in association with EV-D68 infection [20]. However, determination of AFP aetiologies can be challenging, because of the absence of pathogen detection in the CSF. Investigation of AFP cases should include both EV screening of two stool samples collected $\geq 24$ hours apart and $<14$ days after symptom onset [21] and early and quick testing of diverse samples, especially upper respiratory samples, for infectious agents including EVs, to increase the chance to identify a pathogen. In the case of EV-D68 infections, the detection capabilities of the EV-D68 genome of commercial and in-house molecular methods should be assessed. 
Bacteriological and virological investigations performed on clinical specimens collected in the first week of the course of the illness, acute flaccid paralysis case following enterovirus D68 infection, France, 2014

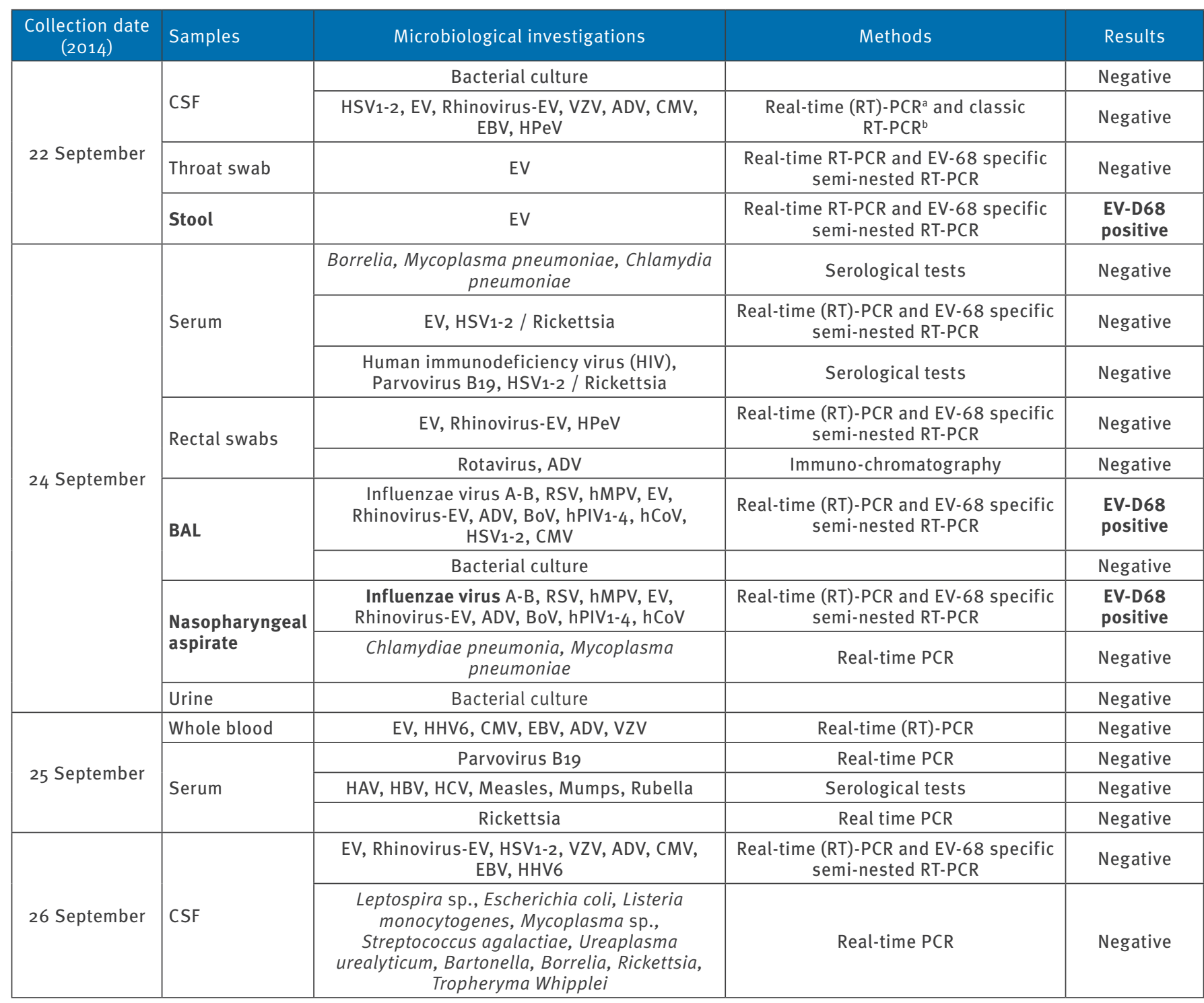

ADV: adenovirus; BAL: bronchoalveolar lavage; BoV: Bocavirus; CMV: cytomegalovirus; CSF: cerebrospinal fluid; EV: enterovirus; EBV: Epstein-Barr virus; HAV: hepatitis A virus; HBV: hepatitis B virus; hCoV: human coronavirus; HCV: hepatitis C virus; HHV: human herpes virus; hMPV: human metapneumovirus; HPeV: human parechoviruses; hPIV: human parainfluenzae virus; HSV: herpes simplex virus; RSV: respiratory syncytial virus; VZV: varicella zoster virus.

a For enteroviruses, a commercial pan-EV RT-PCR was used.

b Classic RT-PCR was used for rhinovirus-EV genome detection [12].

\section{Acknowledgements}

We are grateful to Nathalie Rodde and Gwendoline Jugie for excellent technical assistance in enterovirus genotyping. We thank Jeffrey Watts for revision of the English manuscript. No specific financial support was obtained for the study. The Centre National de Référence des Enterovirus-Parechovirus is supported by an annual grant from the French national public health network (Institut de Veille Sanitaire, InVS).

\section{Authors' contributions}

Mathieu Lang, Nadia Savy, Sarah Maridet and André Labbé were involved in the clinical management of the patient. The virological investigations were performed by Audrey Mirand, Cécile Henquell and Hélène Peigue-Lafeuille. Magnetic resonance imagery was interpreted by Renan Pérignon. Audrey Mirand wrote the first draft of the paper. All authors reviewed the manuscript critically.

\section{Conflict of interest}

None declared. 


\section{References}

1. Midgley CM, Jackson MA, Selvarangan R, Turabelidze G, Obringer E, Johnson D, et al. Severe Respiratory Illness Associated with Enterovirus D68 - Missouri and Illinois, 2014. MMWR Morb Mortal Wkly Rep. 2014;63(36):798-9.

2. National Collaborating Centre for Infectious Diseases (NCCID). Disease Debrief: EV-D68. Winnipeg, Manitoba: NCCID. [Accessed 5 Nov 2014]. Available from: http://www.nccid.ca/ disease-debrief-ev-d68\#Q1

3. Pastula DM, Aliabadi N, Haynes AK, Messacar K, Schreiner T, Maloney J, et al. Acute neurological illness of Unknown etiology in Children - Colorado, August-September 2014. MMWR Morb Mortal Wkly Rep. 2014;63(40):901-2.

4. British Columbia Centre for Disease Control. Emerging Respiratory Virus Bulletin: MERS-CoV, Influenza A(H7 $\left.\mathrm{N}_{9}\right)$ and $\mathrm{A}\left(\mathrm{H}_{3} \mathrm{~N}_{2}\right) \mathrm{v}$, Enterovirus D68. British Columbia: Centre for Disease Control. 4 Oct 2014. Available from: http:// www.bccdc.ca/NR/rdonlyres/88FD3DD4-BEBo-4F29-93Co6093A7AFBD4B/o/Full_ERVUpdate20141004.pdf

5. Renois F, Bouin A, Andreoletti L. Enterovirus 68 in pediatric patients hospitalized for acute airway diseases. I Clin Microbiol. 2013;51(2):640-3. http://dx.doi.org/10.1128/ JCM.02640-12

6. Centers for Disease Control and Prevention. Clusters of acute respiratory illness associated with human enterovirus 68-Asia, Europe, and United States, 2008-2010. MMWR Morb Mortal Wkly Rep. 2011;60(38):1301-4.

7. Piralla A, Baldanti F, Gerna G. Phylogenetic patterns of human respiratory picornavirus species, including the newly identified group C rhinoviruses, during a 1-year surveillance of a hospitalized patient population in Italy. J Clin Microbiol. 2011;49(1):373-6. http://dx.doi.org/10.1128/JCM.01814-10

8. Rahamat-Langendoen J, Riezebos-Brilman A, Borger R, van der Heide R, Brandenburg A, Schölvinck E, et al. Upsurge of human enterovirus 68 infections in patients with severe respiratory tract infections. J Clin Virol. 2011;52(2):103-6. http://dx.doi. org/10.1016/j.jcv.2011.06.019

9. Meijer A, van der Sanden S, Snijders BE, Jaramillo-Gutierrez $G$, Bont L, van der Ent CK, et al. Emergence and epidemic occurrence of enterovirus 68 respiratory infections in the Netherlands in 2010. Virology. 2012;423(1):49-57. http:// dx.doi.org/10.1016/j.virol.2011.11.021

10. Imamura T, Fuji N, Suzuki A, Tamaki R, Saito M, Aniceto R, et al. Enterovirus 68 among children with severe acute respiratory infection, the Philippines. Emerg Infect Dis. 2011;17(8):1430-6.

11. Ikeda T, Mizuta K, Abiko C, Aoki Y, Itagaki T, Katsushima $F$, et al. Acute respiratory infections due to enteroviurs 68 in Yamagata, Japan between 2005 and 2010. Microbiol Immunol. 2012;56(2):139-43. http://dx.doi. $\operatorname{org} / 10.1111 / j .1348-0421.2012 .00411 . x$

12. Savolainen C, Mulders NN, Hovi T. Phylogenetic analysis of human rhinovirus isolates collected during successive epidemic seasons. Virus Res. 2002;85:41-6. http://dx.doi. org/10.1016/S0168-1702(02)00016-3

13. Henquell C, Mirand A, Deusebis AL, Regagnon C, Archimbaud C, Chambon M, et al. Prospective genotyping of human rhinoviruses in children and adults during the winter of 2009-2010. J Clin Virol. 2012;53(4):280-4. http://dx.doi. org/10.1016/j.jcv.2011.10.009

14. Tokarz R, Firth C, Madhi SA, Howie SRC, Wu W, Sall AA, et al. Worldwide emergence of multiple clades of enterovirus 68. J Gen Virol. 2012;93:1952-8. http://dx.doi.org/10.1099/ vir.0.043935-0

15. Centers for Disease Control and Prevention (CDC). Enterovirus D68 in the United States, 2014. Atlanta: CDC. [Accessed 5 Nov 2014]. Available from: http://www.cdc.gov/non-polioenterovirus/outbreaks/EV-D68-outbreaks.html

16. Centers for Disease Control and Prevention (CDC). Health Alert Network. Acute Neurologic illness with focal limb weakness of unknown etiology in children. Atlanta: CDC. 26 Sep 2014. Available from: http://emergency.cdc.gov/han/hano0370.asp

17. Kreuter JD, Barnes A, McCarthy JE, Schwartzman JD, Oberste MS, Rhodes $\mathrm{CH}$, et al. A fatal central nervous system enterovirus 68 infection. Arch Pathol Lab Med. 2011;135(6):793-6.

18. Khetsuriani N, Lamonte-Fowlkes A, Oberste S, Pallansch MA. Centers for disease Control and Prevention. Enterovirus Surveillance-United States, 1970-2005. MMWR Surveill Summ. 2006;55(8):1-20.

19. Perez-Velez CM, Anderson MS, Robinson CC, McFarland EJ, Nix WA, Pallansch MA, et al. Outbreak of neurologic enterovirus type 71 disease: a diagnostic challenge. Clin Infect Dis. 2007;45(8):950-7. http://dx.doi.org/10.1086/521895

20. Shaw J, Welch TR, Milstone AM. The role of syndromic surveillance in directing the public health response to the enterovirus D68 epidemic. JAMA Pediatr. (Forthcoming). 10.1001/jamapediatrics.2014.2628. http://dx.doi.org/10.1001/ jamapediatrics.2014.2628

21. Global Polio Eradication Initiative. Acute flaccid paralysis (AFP) surveillance. Geneva: Global Polio Eradication Initiative. [Accessed 2 Nov 2014]. Available from: http://www. polioeradication.org/Dataandmonitoring/Surveillance.aspx 